NASA TM -84308

NASA Technical Memorandum 84308

Airborne Astronomy Program

Medium Altitude Missions Branch

Preprint Series 003

NASA-TM-84308 19830007992

\title{
Infrared Observations of OB Star Formation in NGC 6334
}

\section{Paul M. Harvey and Ian Gatley}

November 1982

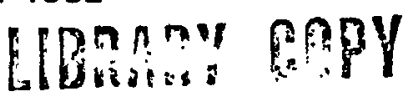

JAN 111983

IANGLEY RESEARCH CENTER LIBRARY, NASA HAMPTON YURGINHA

1

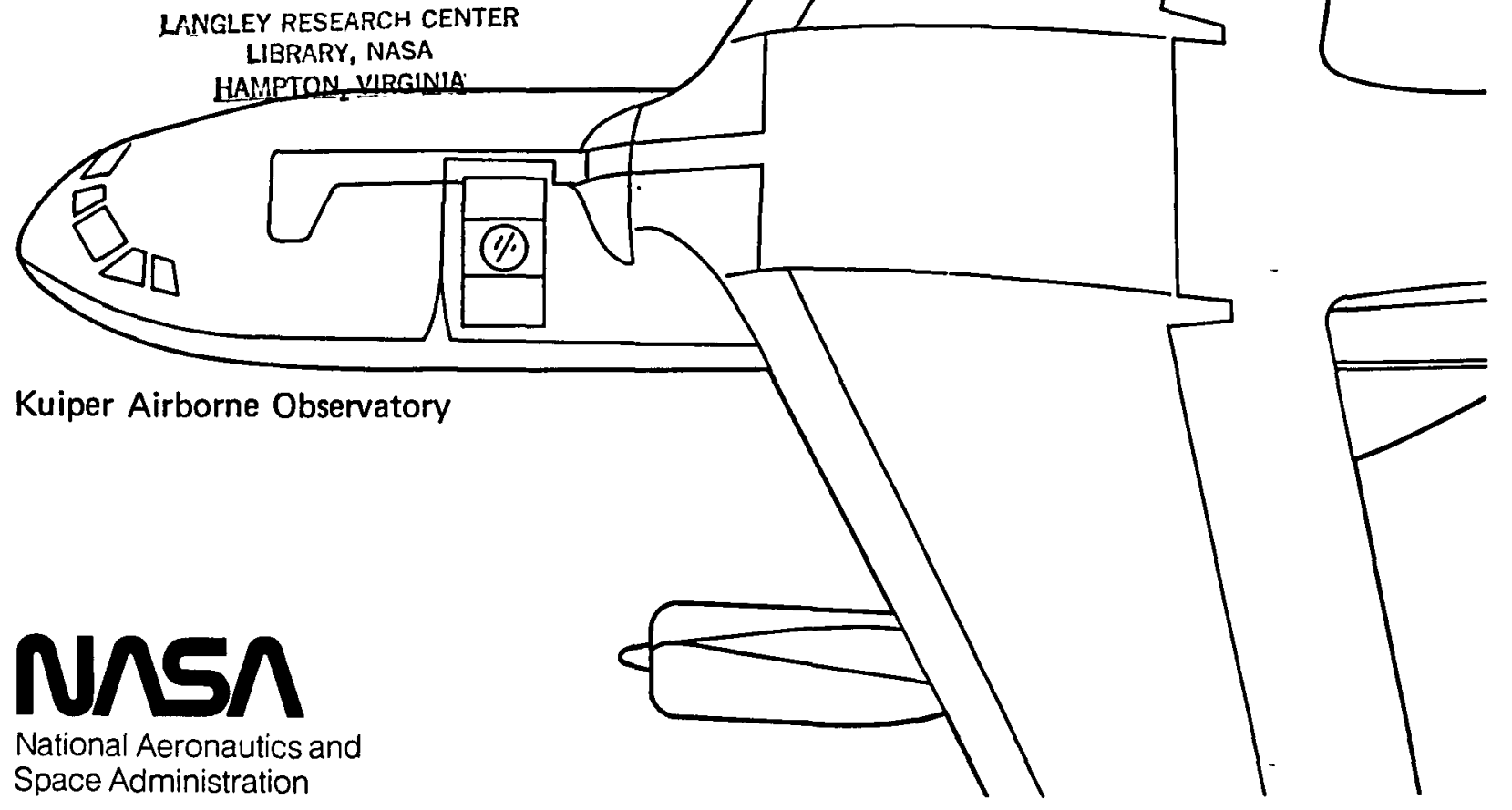




\section{Infrared Observations of OB Star Formation in NGC 6334}

Paul M. Harvey

Ian Gatley, Astronomy Department, Univeristy of Texas and United Kingdom Infrared Telescope

\section{N/SA}

National Aeronautics and

Space Administration

Ames Research Center

Moffett Field, California 94035 
INFRARED OBSERVATIONS OF OB STAR FORMATION IN NGC 6334

by

Paul M. Harvey ${ }^{1,3}$ and Ian Gatley ${ }^{2,3}$

Received September 1982

Accepted for publication in Astrophysical Journal

${ }^{1}$ Astronomy Department, University of Texas.

2 United Kingdom Infrared Telescope.

${ }^{3}$ Visiting Astronomer at the Infrared Telescope Facility which is operated by the University of Hawaii under contract from the National Aeronautics and Space Administration. 
ABSTRACT:

Infrared photometry and maps from 2 to $100 \mu \mathrm{m}$ are presented for three of the principal far-infrared sources in NGC 6334. Each region is powered by two or more very young stars. The distribution of dust and tonized gas is probably strongly affected by the presence of the embedded stars; one of the sources is a blister H II region, another has a bipolar structure, and the third exhibits asymmetric temperature structure. Spherically symmetric models are clearly not applicable to any of the clouds around these objects. The presence of protostellar objects throughout the region: suggests that star formation has occurred nearly simultaneously in the whole molecular cloud rather than having been triggered sequentially from within. 


\section{Introduction}

The NGC 6334 molecular cloud and H II region complex is a large (20 pc) star-forming area with multiple centers of OB star formation. The first infrared studies of this area were made by Emerson, Jennings, and Moorwood (1973) at far-infrared wavelengths $(45-350 \mu \mathrm{m})$ and by Becklin and Neugebauer (1974) at 1.6-20 $\mu \mathrm{m}$. Emerson et al., found three far-infrared sources which they identified with an $\mathrm{OH}$ maser source and with two compact $\mathrm{H}$ II regions mapped by Schraml and Mezger (1969) in $2 \mathrm{~cm}$ continuum emission.. Becklin and Neugebauer (1974) found several near-infrared sources including a compact red object near an $\mathrm{OH}$ maser which they compared to the BN object in Orion, a possible protostar. Subsequently, McBreen et al.. (1979; hereafter MFSW) mapped the region at $\lambda \sim 70 \mu \mathrm{m}$ with higher spatial resolution (1') finding a total of six farinfrared sources. Studies of $\mathrm{CO}$ emission and $\mathrm{H}_{2} \mathrm{CO}$ absorption show that the whole NGC 6334 cloud is collapsing with a $\mathrm{v} \propto \mathrm{r}$ collapse law, and that all the sites of star formation are embedded within this single cloud (Dickel, Dickel and Wilson 1977). Following MFSW, we designate the far-infrared sources by the Roman numerals I-VI; compact near-infrared sources within these regions will then be designated further by the label IRS-1,2,3, etc., as usual (Becklin and Neugebauer, 1974). This nomenclature and the correspondence between sources observed in some of the studies of NGC 6334 is summarized in Table 1.

In this paper we report new infrared observations of regions I, IV and $\mathrm{V}$; these are the three brightest compact far-infrared sources in this large and complicated region. Each contains at least one maser; these are regions of recent star formation (Genzel and Downes, 1977). Sources I and IV coincide with compact $H$ II reglons each of which requires at least one 08 star 
for its excitation. Source $V$ does not appear in the map of Emerson et al. (1973) and may be variable: (MFSW). The areas observed in our study are marked -; in Figure 1 which shows a reproduction of the far-infrared contours of MFSW. The main near-infrared continum peaks which we found within the far-infrared emission regions are also listed in Table 1.

Our study is preliminary in the following sense: because of the large area of the NGC 6334 complex we were not able to observe all suspected sites of star formation. Our measurements of this star formation complex do, however, allow us to address four issues: 1) The nature of the newly formed stars in the observed regions, 2) the distribution of density, temperature and luminosity around these young objects, 3). the possibility that the turnon of the young stars has strongly "acted-back" on the local interstellar medium and 4) the mode of onset of star formation, for example by spiral density wave or sequential formation within the cloud.

In $\S$ II we describe the instrumentation and observations. In $\$ \S$ III, IV and $V$ we present the results of observations of the regions NGC 6334 - I, IV and $V$ respectively and discuss interpretation of the observations specific to the individual sources. In $\delta$ VI we discuss the large scale structure of the NGC 6334 complex and the implications of this study on our understanding of $O B$ star formation.

\section{Instrumentation and Observations}

Observations at wavelengths 2-30 $\mu \mathrm{m}$ were made in 1981 June on the NASA IRTF and UKIRT telescopes on Mauna Kea, Hawail. The standard facility infrared photometers were used with the instrumental parameters listed in Table 2 . All photometric measurements had statistical uncertainties less than $5 \%$ unless 
otherwise noted. The statistical uncertainties of the mapping observations are described in the appropriate figure captions. In all cases, the photometric observations were obtained at the positions of peak $20 \mu \mathrm{m}$ surface brightness as measured with an 8 " beam.

The 50 and $100 \mu \mathrm{m}$ maps were made in 1979 August on the Kuiper Airborne Observatory flying out of Hickam Field, Hawail, at an altitude of $12.5 \mathrm{~km}$. The photometer described by Harvey (1979) was used with the instrumental parameters shown in Table 2. The observations were calibrated relative to S 140-IR (Harvey et al., 1978). The statistical uncertainties in the maps are described in the figure captions.

The observed flux densities were converted to true surface brightnesses based on measured beam profiles. The contour spacing of the maps was chosen to be some reasonable multiple of the noise level. For maps where the signalto-noise was large, this leads to a narrow contour spacing on the sky. For regions which appeared to be point-like, the compact area was drawn crosshatched.. For sources which were definitely resolved, all formal contours are shown in order to convey information which appears to be present in the raw data.

III. NGC $6334-$ I

A. 50-100 um Maps - Luminosity and Large Scale Structure

The results at 50 and $100 \mu \mathrm{m}$ are shown in Figures $2 \mathrm{a}-\mathrm{d}$. Figures $2 \mathrm{a}$ and b show the surface brightness contours at 50 and $100 \mu \mathrm{m}$; Figure $2 \mathrm{c}$ shows the dust temperature derived by assuming thermal emission from dust with an emissivity $\varepsilon \propto \lambda^{-1}$; and Figure $2 \mathrm{~d}$ shows the dust optical depth at $100 \mu \mathrm{m}$ derived from the dust temperature above and the observed $100 \mu \mathrm{m}$ flux density. The absolute uncertainties in dust temperature and optical depth are typically $\pm 10 \mathrm{~K}$ 
and \pm a factor of 2 respectively. The relative uncertainties within each map, however, are considerably better since the two wavelengths were observed simultaneously. These uncertainties, which bear on the reality of the structure in the maps of $T_{d}$ and $\tau$, are $\pm 1.5-2 \mathrm{~K}$, and $\pm 20 \%$ in $\tau$ for all the. sources observed in NGC 6334, regardless of the assumed wavelength dependence of the dust opacity or the absolute calibration uncertainties.

The total flux density at 50 and $100 \mu \mathrm{m}$ contained within the mapped area is Iisted in Table 3 together with the implied luminosity, assuming a spectrum of the form $V B_{V}$ fit to the 50 and $100 \mu \mathrm{m}$ flux densities. The total integrated flux densities observed in this study agree within the mutual uncertainties with those observed by MFSW with a larger beam. Therefore, Figure 2 contains all the significant luminosity in this region. Figures $2 a$ and $2 b$ show that. the emission is strongly peaked at the position of the 2-30 $\mu \mathrm{m}$ compact source discussed below. There is, however, diffuse emission extended to the northwest and west at 50 and $100 \mu \mathrm{m}$ and to the north at $100 \mu \mathrm{m}$ only. Figures $2 \mathrm{c}$ and $\mathrm{d}$ show that there is a large gradient in temperature and in dust optical depth in the diffuse emission. The dust is hotter and much less dense to the northwest and west than to the north.

This structure is similar to that inferred from other observations; the $400 \mu \mathrm{m}$ and $1 \mathrm{~mm}$ maps of Gezari (1982) and Cheung et al. (1978) show a large column density of cold dust 2 ' north of the present peak. Low resolution radio continuum maps (Schraml and Mezger 1969) and optical photographs show an extended, diffuse region to the northwest suggesting low attenuation in that direction. A diffuse H II region $1^{\prime}$ northwest of the far-infrared peak (Rodriguez, Canto and Moran 1982; hereafter RCM) is probably responsible for the extension of the higher dust temperature contours in that direction. The contribution of this $\mathrm{H}$ II region to the total luminosity can be crudely 
estimated from our maps as about $30 \%$ of the total luminosity or L $\sim 4 \times 10^{4}$ $\mathrm{L}_{0}$

\section{B. 2-30 $\mu \mathrm{m}$ Maps - Sma1l Scale Structure}

Figures $2(\mathrm{e})$ and $2(\mathrm{f})$ show $20 \mu \mathrm{m}$ maps and Figure $2(\mathrm{~g})$ a $30 \mu \mathrm{m}$ map of NGC 6334-I. The energy distribution at the position of highest $20 \mu \mathrm{m}$ surface brightness as measured with an 8 " beam is shown in Figure 3; for comparison, the integrated $20 \mu \mathrm{m}$ and $30 \mu \mathrm{m}$ fluxes are also plotted. The peak position in Figures $2(\mathrm{e})-(\mathrm{g})$ is that of the well-known protostar, NGC 6334-I-IRS-1 (Becklin and Neugebauer 1974). An additional search at $20 \mu \mathrm{m}$ of a region 50" square centered 120" north in the cool dense cloud failed to find any source brighter than $20 \mathrm{Jy}(3 \sigma)$.

Figures $2 \mathrm{e}-\mathrm{g}$ show a variety of structure around the near-infrared source. At high resolution, the source is extended to the northwest, or probably double with a component separation of about 6". The northwest component is noticeably cooler than the central peak as shown by the higher relative flux density at $30 \mu \mathrm{m}$. The central source is extended to the east and southeast but does not appear to be significantly different in 20-30 $\mu \mathrm{m}$ color temperature in this area. The emission at both 20 and $30 \mu \mathrm{m}$ drops off sharply to the southwest.

To the east and southeast, the high-resolution $20 \mu \mathrm{m}$ and $30 \mu \mathrm{m}$ maps $100 \mathrm{k}$ similar to the radio map of RCM (Source F). RCM suggested that the radio source is a "nozzle" created by the location of an ionizing star in a strong density gradient increasing from east to west. The coincidence of the 20/30 $\mu \mathrm{m}$ emission peak with the radio peak, the similarity in extension to the southeast, and the lack of a temperature gradient suggest that in this region we are seeing heated dust associated with the compact $\mathrm{H}$ II region around a star near the radio surface-brightness peak. If (as RCM suggest) 
the sharp drop in radio flux to the west, northwest, and southwest of IRS-1 occurs because a strong density. gradient creates an ionization limited boundary, then the cool NW lobe in the infrared may represent another luminosity source embedded in the high density material to the west. This source, designated IRS-2, evidently does not emit a significant amount of ionizing radiation.

Two alternative possibilities for IRS-2 are that: (1) it represents.a temperature or column density enhancement in the dust heated by IRS-1; or (2) it is in fact the position of a single luminosity source whose lonizing flux is restricted to an unobservably small volume in the vicinity of IRS-2 but, because of the decreasing density toward IRS-1, is able to Ionize the region of IRS-1. Both these models, however, require very contrived geometries and density structure. The first model would require the density gradient from SE to NW to first increase rapidly at the NW boundary of the radio-continuum source and then decrease immediately beyond that point sufficiently to allow non-ionizing heating photions to be absorbed preferentially at the position of IRS-2 rather than much closer to the boundary of the radio-continuum source. The second model would require a very high density around IRS-2 except in a "long, narrow" tube in the direction of IRS-1. The geometrical appearance of the radio source is perfectly consistent with models of $0-B$ stars embedded in a density gradient (Icke, Gatley and Israel 1980). The position of the source of ionization in such a case would be close to, but slightly east of the radiocontinuum peak. Therefore, our maps are most consistent with a two-source model for IRS-1 and 2 .

There are both $\mathrm{H}_{2} \mathrm{O}$ and $\mathrm{OH}$ maser emission sources in the vicinity of IRS-1. The $\mathrm{H}_{2} \mathrm{O}$ maser position has been measured with only $30^{\prime \prime}$ accuracy. The OH maser position in this area reported by RCM is not located near any observed $20 / 30 \mu \mathrm{m}$ 
source or extended region. Rather, at that position (about 20" south of IRS-1) the observed flux density $F_{\nu}<7 \mathrm{Jy}(3 \sigma)$ at $20 \mu \mathrm{m}$, or $1 / 50$ that of IRS-1. The OH maser position measurements at several other velocities (Raimond and Eliasson 1969) do, however, show one velocity component which is near the infrared peak as noted previously by $\mathrm{BN}$.

\section{Nature of the Sources in NGC 6334-I}

The total luminosity of NGC 6334-I, observed in the far infrared, is $1.5 \times 10^{5} \mathrm{I}_{0^{\prime}}$ (Table 3 ). This luminosity is, of course, the sum of the 1uminosities of the individual embedded sources. It is straightforward to deduce the relative contributions of the embedded sources. These values are given in Table 4, and are deduced as follows. There are four luminous compact sources found within NGC 6334-I: the protostar IRS1; the $\operatorname{coo} 120-30 \mu \mathrm{m}$ source IRS-2, 6" to the northwest; the $2 \mu \mathrm{m}$ peak associated with an extension of the $20 \mu \mathrm{m}$ contours $15^{\prime \prime}$ to the east, designated IRS-3; and the H II region 60 " to the northwest, designated IRS-4 (source "E" of RCM). Other $2 \mu \mathrm{m}$ sources in the map of Becklin and Neugebauer (1974) have no $20 \mu \mathrm{m}$ counterparts. They probably are low luminosity stars or reflection nebulosity. IRS-1 is plausibly the major source of Iuminosity in the region because it coincides with the peak in surface brightness at every wavelength. IRS-2 has a spectrum which rises more steeply than that of IRS-1 between 20 and $30 \mu \mathrm{m}$, is one-third as bright as IRS-1 at $30 \mu \mathrm{m}$, and has a deeper silicate absorption at $\lambda \sim 10 \mu \mathrm{m}$; therefore, the luminosity of IRS-2 is at least onethird that of IRS-1. The contribution of IRS-3 is clearly less: this source is fainter at $20 \mu \mathrm{m}$ and has a bluer energy distribution. Based on its brightness at $2 \mu \mathrm{m}$ and $20 \mu \mathrm{m}$ relative to IRS-1, the luminosity of IRS-3 is approximately one-tenth of that of IRS-1. The luminosity of IRS-4 is directly 
measured because it is spatially resolved from the other sources in the farinfrared.

In Table 4, the infrared luminosity and radio fluxes are used separately to deduce the ZAMS stellar type implied for the compact sources. Both IRS-1 and IRS-2 have the property that the ultraviolet flux which sustains the ionization of the H II region is considerably less than that provided by a ZAMS star of the measured luminosity. Measurements of $B \alpha$ (Simon et al., 1981) show that the H II region is not optically thick at $6 \mathrm{~cm}$. Absorption of Lyman continuum photons by dust in the dense circumstellar environment, or a pre-main sequence nature for the exciting stars are implied (e.g., Wynn-Williams 1982); In either case, these objects are very young.

IRS-3 is probably an embedded B star. IRS-4 is less luminous in the farinfrared than would be expected from the radio flux. It is likely that some of the non-ionizing radiation escapes absorption by dust local to the source. This implies a lower interstellar density at IRS-4 than at IRS-1 and 2; this source is rather more evolved. The escaping luminosity is probably absorbed at greater distances, and contributes to the diffuse far-infrared emission observed by MFSW, Hoffmann, Frederick and Emery (i971) and 0Ithof and Van Duinen (1973).

IV. NGC 6334-IV

A. 50-100 $\mu \mathrm{m}$ Maps - Luminosity and Large Scale Structure

The far-infrared maps of surface brightness, dust temperature and dust optical depth are shown in Figures $4 a-d$, and the integrated fluxes are listed in Table 3. The total integrated flux densities agree well with that observed by MFSW, implying that our map probably includes all significant sources of luminosity. 
This region is the most extended and least centrally peaked in the farInfrared of the three observed in our study. The 50 and $100 \mu \mathrm{m}$ maps have a slight luminosity peak at the center of the map and a secondary peak about $I^{\prime}$ southeast. The temperature map in Figure $4 \mathrm{c}$ shows complicated structure with local temperature maxima $1^{\prime}$ north and south of the central luminosity peak and at the secondary southeastern luminosity peak. This asymmetry can also be seen qualitatively in the greater north-south extent of the $50 \mu \mathrm{m}$ emission relative to the $100 \mu \mathrm{m}$ emission. The dust optical depth appears highest along an east-west bar through the center. This central structure is consistent with the higher resolution $20 \mu \mathrm{m}$ maps and will be discussed below. The southeast lobe is not associated with any known optical or radio continuum feature.

\section{B. 2-30 $\mu \mathrm{m}$ Maps - Smal1 Scale Structure}

Figure $4(\mathrm{e})$ shows a $20 \mu \mathrm{m}$ map of NGC 6334-IV covering an area of $2^{\prime} \times 3^{\prime}$ with 8" resolution. Four bright sources, labeled IRS-1, 2, 3, 4 are seen together with some diffuse emission. The energy distributions of these four sources are shown in Figure 3. Figure $4(\mathrm{f})$ shows a $2.2 \mu \mathrm{m}$ map covering an area $2^{\prime} \times 2^{\prime}$ with $10^{\prime \prime}$ resolution. Sources IRS-1 and 3 are seen in this map; there is a $2 \mu \mathrm{m}$ emission ridge associated with the western edge of the $20 \mu \mathrm{m}$ source IRS-4; and IRS-2 is not seen. The two further sources in Figure 4(f), IRS-5 and 6, are visible stars seen on the POSS print. There is also.considerable extended emission west of the dotted line in Figure $4(f)$. This is presumably reflection nebulosity. Its presence limits the accuracy of the deconvolution of the fluxes in the $2.2 \mu \mathrm{m}$ map to about $30 \%$.

IRS-2 is extended, being about $12^{\prime \prime}$ in diameter. It is coincident with a ring-like radio source (source " $\mathrm{A}$ " of $\mathrm{RCM}$ ), but shows no such structure in the infrared. IRS-3 coincides with an $\mathrm{H}_{2} \mathrm{O}$ maser (Rodriguez et al., 1980). 
The diffuse sources IRS-1 and 4 have no radio continuum or maser counterpart, but IRS-1 is associated"with faint optical nebulosity on the POSS red print.

\section{Nature of the Sources in NGC 6334-IV}

The total Iuminosity of all the objects embedded in the region, NGC 6334-IV is $\mathrm{L} \sim 2 \times 10^{5} \mathrm{~L}_{\odot}$ (Table 3). The luminosity required for a ZAMS star to produce the $\mathrm{H}$ II region " $\mathrm{A}$ " $(\mathrm{RCM})$ is $\mathrm{L} \sim 8 \times 10^{4} \mathrm{~L}_{\odot^{\circ}}$ Therefore, the exciting star for this $\mathrm{H}$ II region and IRS -2 must be an important source of luminosity in the region. Another major source of luminosity must be IRS-3. This conclusion follows because, at 20 and $30 \mu \mathrm{m}$, IRS -3 is 50 to 75 percent brighter than IRS-2. Furthermore, the $10 \mu \mathrm{m}$ silicate absorption feature (Figure 3 ) is considerably deeper for IRS-3 than IRS-2, suggesting substantial absorption of energy, and reradiation at longer wavelengths. We conclude that the exciting stars for IRS-2 and 3 probably supply most of the luminosity seen in the far-infrared. IRS -1 and 4 contribute less than $15 \%$ each to the total, or $\mathrm{L} \sim 4 \times 10^{4} \mathrm{~L}_{\odot}$. Table 5 summarizes these estimates of individual source luminosities.

IRS-3 has properties typical of galactic protostars (Wynn-Williams 1982).' Like the source NGC 6334-I-IRS-2, described above in $\S$ III(C), a ZAMS star of this luminosity should ionize far more gas than is presently deduced from the radio flux.

IRS-2 is a bright radio source (RCM) in which the infrared and radio morphology are strikingly different; the infrared source is centrally concentrated, but the radio source is ring-like. The far-infrared temperature peaks north and south of IRS-2 (Figure $4(\mathrm{c})$ ) while the dust column density peaks in an east-west bar through IRS-2 and 3. The diffuse sources IRS-1 and 4 are located north and south of IRS-2 and 3 in the regions of the temperature 
maxima. An attractive hypothesis for this somewhat unusual situation is that this whole region is a bipolar nebula excited by IRS-2 and/or IRS-3.

In this picture, IRS-2 and 3 are surrounded by a dense ring or bar of gas and dust, extended east-west. The double-lobes are IRS-1 and 4, 11luminated by radiation from the central star(s) which propagates further in the north and south direction because the density is lower. This model predicts large polarization of the $2.2 \mu \mathrm{m}$ emission from the lobes and also explains the lack of bright near-infrared point sources in IRS-1 and 4. This is similar to the situation found in the bipolar nebula S106 (Harvey et al., 1982), although NGC 6334-IV is both larger and more luminous than S106. We may compare the two objects quantitatively by assuming that the spatial extent at $20 \mu \mathrm{m}$ is defined by the region over which the dust is hotter than $\mathrm{T} \sim 100 \mathrm{~K}$. In S106, Gehrz et al. (1982) have argued that the dust emitting at 10-20 $\mu \mathrm{m}$ is heated predominantly by trapped Lyman- $\alpha$. If we scale the two objects by Ly-a luminosity, $L_{\alpha}^{0: 25}$ and size, $R^{-0.5}$ to compare the relative dust temperatures in the lobes, we find both bipolar nebulae are of equivalent extent. Like S106, the dust temperatures implied by the 10-30 $\mu \mathrm{m}$ flux densities in the lobes of NGC 6334-IV are too high to be explained by heating by the geometrically diluted stellar luminosity; they can probably be explained by heating from trapped $L y-\alpha$ radiation in the $H$ II region (RCM).

V. NGC 6334-V

A. 50-100 $\mu \mathrm{m}$ Maps - An Unresolved Source

A $1.5^{\prime} \times 2^{\prime}$ area (Dec. $\times$ R.A.) was mapped; one unresolved source was found. Figures $5 \mathrm{a}$ and $\mathrm{b}$ show the results in terms of the source profile relative to the beam profiles. These profiles imply an upper limit to the 
source diameter of $20^{\prime \prime}$ at $50 \mu \mathrm{m}$ and $25^{\prime \prime}$ at $100 \mu \mathrm{m}$. The total far-infrared luminosity of this object is $\mathrm{L} \sim 6.5 \times 10^{4} \mathrm{~L}_{\ominus}$ (Table 3). This estimate is a factor of 2-3 lower than that of MFSW, presumably because their calculation included the extended, low-surface-brightness emission around the compact source. It is difficult to determine how much of that extended emission is powered by the central star of our compact source and how much by other distant early-type stars. We will assume a total luminosity for the object(s) embedded in the core of this region to be $\mathrm{L}=10 \pm 4 \times 10^{4} \mathrm{~L}_{0}$. There are no known compact radio-continuum sources in this region with which to compare the far-infrared source position. There is an $\mathrm{H}_{2} \mathrm{O}$ maser which is probably associated with this object (Moran and Rodriguez 1980), but the positional uncertainty of $\pm 30^{\prime \prime}$ is comparable to the far-infrared source size.

\section{B. 2-30 $\mu \mathrm{m}$ Maps - Smal1 Scale Structure}

Figures $5(\mathrm{c})$ and $5(\mathrm{~d})$ show maps of NGC $6334-\mathrm{V}$ at $20 \mu \mathrm{m}$ with 8 " and $4 "$ resolution, and Figure $5(\mathrm{e})$ shows a $30 \mu \mathrm{m}$ map with 4 " resolution. Figure $5(f)$ shows right ascension scans through the position of peak $20 \mu \mathrm{m}$ surface brightness at 10 and $20 \mu \mathrm{m}$ with 8 " resolution, and Figure 5(g) shows declination scans at 10, 20 and $30 \mu \mathrm{m}$ with 4 " resolution. Table 6 gives $\mathrm{K}$ and $\mathrm{L}$ measurements of six sources found in a search for candidates for the exciting $\operatorname{star}(s)$.

The 20 and $30 \mu \mathrm{m}$ maps show an east-west ridge of emission. The east end of this ridge has a higher color temperature than the west. The northern side of this ridge is unresolved, but the southern side is successively more extended at longer wavelength'; showing that the grain temperature falls to the. south. All of the near-infrared sources in this region (Table 6) are red in 
$\mathrm{K}-\mathrm{L}$. The reddest of these, $\$ 3$, is coincident within the observational errors with the position of the ridge. Both the $20 \mu \mathrm{m}$ ridge and $2 \mu \mathrm{m}$ source IRS-3 probably are the same source as found recently by Fischer et al. (1982). Our measurements of this object from 10-20 $\mu \mathrm{m}$ with an 8 " beam agree quite well with these of Fischer et al., with a $5^{\prime \prime}$ beam given the observed source extent. Our 2.2, 3.5, and $8.7 \mu \mathrm{m}$ photometry, however, disagree substantially. The most likely reason for this is that the object is extended at all observed wavelengths (this paper and Fischer et al.) and there are strong color gradients. In addition, the source in Table 6 labelled IRS-4 may be part of this same object. It is likely that high-pointing-accuracy mapping at several wavelengths from 1.6 to $20 \mu \mathrm{m}$ will be required to resolve this problem.

\section{Nature of the Source(s) in NGC 6334-V}

The present observations are not definitive of the nature of NGC 6334-V, but provide some interesting constraints. If the source is heated by an embedded star, its total luminosity, obtained by integration of the measured fluxes from 10 to $100 \mu \mathrm{m}$, is $\mathrm{L} \leq 6 \times 10^{4} \mathrm{~L}_{0^{\circ}}$ A ZAMS star of this luminosity would produce a radio flux far in excess of that observed (RCM). This is typical of galactic protostars (Wynn-Williams 1982; compare also the discussions of NGC $6334-$ I-IRS-2 and IV-IRS-3 above).

The complicated morphology observed at 10, 20 and $30 \mu \mathrm{m}$ is, however, not supportive of this simple picture, a problem compounded by the lack of multicolor shorter wavelength maps. An alternative model might, for example, be constructed under the assumption that the ridge is excited by two stars situated along an east-west line. Even in this case, the lack of detectable radio emission remains suggestive, however, of a deficiency of Lyman continuum 
photons. The fact that the object is extended east-west and shows a definite north-south temperature:"gradient suggests a third-hypothesis for the"source structure, that the dust is externally heated. Other examples of such objects are the ionization fronts in OMC-1 (Becklin et al. 1976) and MI7 (Gatley et al. 1979). In such a case, however, one would expect to see an extended radio continuum source, contrary to the observations of RCM. Further observations of this target are clearly required.

VI. Star Formation in NGC 6334

A. Density Structure of the H II Regions

The structure of the H II regions NGC $6334-$ I-IRS-1 and 4 is consistent with the interpretation that the ionizing stars are located in a strong density gradient which allows the Strömgren radius to extend much farther on one side than on the opposite side (Icke, Gatley and Israel 1980). This situation appears to be a very common feature of most H II regions which have been carefully mapped in the infrared and radio (e.g., Zuckerman 1973; Gatley et a1. 1979; Hyland et al. 1980). It is not yet clear, however, whether this phenomenon is causally related to the onset of star formation or is simply the result of evolutionary effects which exaggerate pre-existing density inhomogeneities. The large number of such blister H II regions observed has led to the suggestion that $O B$ stars may preferentially form in density gradients (Icke 1979).

The H II region NGC 6334-IV-IRS-2 is, however, not as easily described by the blister model. Rather, our observations can be interpreted as implying the presence of a higher density disk or bar in an east-west plane with lower density above and below this disk. Bodenheimer, Tenorio-Tagle, and 
Yorke (1979) have suggested that this may be a special case of the "blister" model; if the central star is, in fact, located near the point of maximum density, then a two-sided blister results. This structure also suggests that angular momentum and/or magnetic fields have played a significant role in the development of the $H$ II region. In fact, the alignment of IRS-2 and 3 in this region with the dust "bar" suggests that they may be a proto-double star which has condensed in the plane of the larger scale disk-like cloud.

\section{B. Protostars and Star Formation Triggers}

The NGC 6334 region contains at least three "protostars" in the terminology of Wynn-Williams (1982). ${ }^{1}$ These are NGC 6334-I-IRS-2, IV-IRS-3 and V-IRS-3. Two of the major far-infrared sources (II and III of MFSW) were not mapped in

IAlthough the term "protostar" is subject to various interpretations, WynnWilliams has enumerated several characteristics which suggest the extreme youth of some infrared sources. These are (1) most of the luminosity.emitted longward of $2 \mathrm{\mu m}$, (2) association with molecular clouds, (3) heavy dust extinction, (4) association with $\mathrm{OH}$ or $\mathrm{H}_{2} \mathrm{O}$ masers, and (5) lack of an $\mathrm{H}$ II region detectable in the radio-continuum.

our study; thus, it is likely that more protostars will be found in NGC 6334 . This area has the greatest number of centers of recent $O B$ star formation of any H II complex yet surveyed. This fact leads us to ask what has caused the recent and prolific burst of star formation.

Cheung et al. (1980), and Moran and Rodriguez (1980) have proposed that the central regions of the NGC 6334 complex may be older than the outer reglons, suggesting that the center of the cloud formed stars first, which, then triggered star formation in the outer regions. More recently, RCM noted that one of the more evolved H II regions, their source $E$, is the source farthest from the center in the northwest direction. Therefore, they argue, the 
simplest view of self-induced sequential star formation cannot explain the overall structure of the NGC 6334 complex. Our data provide further problems for such a simple model because of the presence of both very young "protostars" and H II regions throughout the molecular cloud.

Theoretical considerations of evolution and expansion time scales suggest that "protostars" and very compact H, II regions are probably less. than a few $\times 10^{4}$ years old. Although it is possible that the collapse of three or more sub-clouds in NGC 6334 occurred randomly to this degree of simultaneity, it seems more likely that some mechanism has triggered the collapse of the cloud over a large area. The extent of the NGC 6334 cloud over which such recent star formation is observed is $\sim 10 \mathrm{pc}$. Therefore if the current observed star formation at many locations were externally triggered from a point, the trigger would have to have a phase velocity $\mathrm{V} \sim 10 \mathrm{pc} / 3 \times 10^{4}$ years $=300 \mathrm{~km} \mathrm{~s} \mathrm{~s}^{-1}$.

This value is unacceptably large unless the current placement of young objects, in fact, reflects the surface of equal phase of the trigger. The data clearly favor a picture in which star formation was initiated throughout the cloud simultaneously. The distance and radial velocity of the molecular cloud (Dickel et al. 1977) place it within the Carina-Sagittarius. spiral arm (Georgelin and Georgelin 1976; Neckel 1978). Although other possibilities exist, the simplest explanation for the widespread star formation is that NGC 6334 is a segment of a spiral arm, triggered into star formation by the passage of a spiral density wave. 


\section{ACKNOWLEDGMENTS}

We thank the staffs of the Kuiper Airborne" Observatory, the Infrared Telescope Facility and the United Kingdom Infrared Telescope. We are grateful to R. Koehler, B'. Schaefer, and H. A. Thronson, Jr., for their help in obtaining the observations. We also thank J. Emerson and an anonymous referee for a number of comments which improved this paper. This work was supported by grants NGR 03-002-390 to the University of Arizona, NAG 2-67 to the University of Texas, and by the Royal Observatory of Edinburgh. 
TABLE 1

Nomenclature of Infrared Sources in NGC 6334

\begin{tabular}{|c|c|c|c|c|c|c|c|c|c|c|c|}
\hline $\begin{array}{l}\text { (1) } \\
\text { Large Area } \\
\text { aar-IR Source 非 } \\
\text { (MISSW) }\end{array}$ & $\begin{array}{c}\text { (2) } \\
\text { Schraml \& Mezger } \\
\text { Source \# }\end{array}$ & $\begin{array}{l}\text { (3) } \\
\text { Emerson et al. } \\
\text { Source it }\end{array}$ & $\begin{array}{l}(4) \\
\text { Near-IR Components } \\
\text { (This Paper) }\end{array}$ & R.A. & $\begin{array}{r}\text { Po } \\
(1950) \\
17 \mathrm{~h}\end{array}$ & $\begin{array}{l}\text { 5) } \\
\text { itio } \\
\text { De }\end{array}$ & $\begin{array}{l}\text { n } \\
\text { c. } \\
-35\end{array}$ & $\lambda_{0}(\mu \mathrm{m})$ & & \multicolumn{2}{|c|}{$\begin{array}{l}(6) \\
\text { Compact Radio- } \\
\text { Continuum } \\
(\mathrm{RCM})\end{array}$} \\
\hline $\mathrm{I}-\mathrm{N}$ & None & None & None $>20 \mathrm{Jy} @ 20 \mu \mathrm{m}$ & $17^{\mathrm{m}}$ & 32.5 & $42^{\prime}$ & $30 "$ & (c) $400^{\circ}$ & $\mu \mathrm{m}$ & . & \\
\hline I & Low-Level Wings & 14 (North) & $\begin{array}{r}\text { IRS-I-1. } \\
2 \\
3 \\
4\end{array}$ & $\begin{array}{l}17 \\
17 \\
17 \\
17\end{array}$ & $\begin{array}{l}32.30 \\
31.95 \\
33.7 \\
30\end{array}$ & $\begin{array}{l}44 \\
44 \\
44 \\
43\end{array}$ & $\begin{array}{l}06 \\
02 \\
05 \\
20\end{array}$ & $\begin{array}{r}20 \\
20 \\
2 \\
50\end{array}$ & $\begin{array}{l}\mu \mathrm{m} \\
\mu \mathrm{m} \\
\mu \mathrm{m} \\
\mu \mathrm{m}\end{array}$ & $\begin{array}{l}\text { F } \\
\text { E }\end{array}$ & \\
\hline II & $351.34+0.6$ & 14 (Center) & & & & & & & & D & , \\
\hline III & $351.31+0.6$ & 14 (Center) & & & & & & . & & C & N \\
\hline IV & $351.2+0.6$ & 14 (South) & $\begin{array}{r}\text { IRS-IV-1 } \\
2 \\
3 \\
4 \\
5 \\
6\end{array}$ & $\begin{array}{l}16 \\
16 \\
16 \\
16 \\
16 \\
17\end{array}$ & $\begin{array}{l}57.2 \\
58.05 \\
56.50 \\
57.1 \\
53.5 \\
00.2\end{array}$ & $\begin{array}{l}51 \\
51 \\
51 \\
52 \\
51 \\
52\end{array}$ & $\begin{array}{l}05 \\
43 \\
53 \\
15 \\
23 \\
14\end{array}$ & $\begin{array}{r}20 \\
20 \\
20 \\
20 \\
2 \\
2\end{array}$ & $\begin{array}{l}\mu \mathrm{m} \\
\mu \mathrm{m} \\
\mu \mathrm{m} \\
\mu \mathrm{m} \\
\mu \mathrm{m} \\
\mu \mathrm{m}\end{array}$ & . & 1 \\
\hline $\mathrm{v}$ & None & None & IRS-V $-3 / 20 \mu \mathrm{m}$ Ridge & 16 & 36.05 & 54 & 44 & 20 & $\mu \mathrm{m}$ & & \\
\hline
\end{tabular}

${ }^{a}$ Gezari (1982).

becklin and Neugebauer (1974). 
TABLE 2

Instrumental Parameters

\begin{tabular}{|c|c|c|c|c|c|}
\hline $\begin{array}{c}\lambda \\
(\mu \mathrm{m})\end{array}$ & Telescope & $\begin{array}{l}\text { Aperture } \\
\text { Sizes } \\
\text { (arcsec) }\end{array}$ & $\begin{array}{l}\text { Choppér } \\
\text { Amplitude } \\
\text { (arcsec) }\end{array}$ & $\begin{array}{c}\text { Positional } \\
\text { Accuracy } \\
\text { (arcsec) }\end{array}$ & $\begin{array}{l}\text { Absolute } \\
\text { Calibration } \\
\text { Uncertainty }\end{array}$ \\
\hline 2.2 & UKIRT $3.8-\mathrm{m}$ & $10^{\prime \prime}$ & $55^{\prime \prime}$ & $7^{\prime \prime}$ & $\pm 10 \%$ \\
\hline 3.5 & 11 & " & " & 11 & $"$ \\
\hline 8.7 & IRTF $3.0-\mathrm{m}$ & $8^{\prime \prime}$ & $40^{\prime \prime}$ & $\leq 1^{\prime \prime}$ & $"$. \\
\hline 9.7 & in & 8" & " & & " \\
\hline 10.0 & $"$ & $4^{\prime \prime}, 8 "$ & " & $"$ & $"$ \\
\hline 10.3 & 19 & $8^{\prime \prime}$ & " & 1 & $\because " \because$ \\
\hline 11.6 & "i" & $"$ & $" \therefore$ & :" & $"$ \\
\hline 12.5 & $"$ & . " ! & 11 & & $"$ \\
\hline 20 & " & $4^{\prime \prime}, 8^{\prime \prime}$ & "1. . & & $20 \%$ \\
\hline 33 & $"$ & $4 ", 8 "$ & $"$ & " & $\therefore 25 \%$ \\
\hline 50 & $0.9-m$ & $35^{\prime \prime}$ & $8^{\prime}$ & $10^{\prime \prime}$ & " \\
\hline 100 & $"$ & $40^{\prime \prime}$ & $"$ & $10^{\prime \prime}$ & $"$ \\
\hline
\end{tabular}


TABLE 3

Integrated Fluxes and Total Luminosities

\begin{tabular}{|c|c|c|c|c|}
\hline \multirow[t]{2}{*}{ Far-IR \# } & \multirow{2}{*}{$\begin{array}{l}\text { Integrate } \\
20 \mu \mathrm{m}\end{array}$} & \multicolumn{2}{|c|}{ Flux Density ${ }^{a}(\mathrm{Jy})$} & \multirow{2}{*}{$\begin{array}{c}\text { Total } \\
\text { Luminosity }\left(L_{\odot}\right)\end{array}$} \\
\hline & & $50 \mu \mathrm{m}$ & $100 \mu \mathrm{m}$ & \\
\hline I & $9 \times 10^{2}$ & $2 \times 10^{4}$ & $3 \times 10^{4}$ & $1.5 \times 10^{5}$ \\
\hline IV & $9 \times 10^{2}$ & $2 \times 10^{4}$ & $4 \times 10^{4}$ & $2.0 \times 10^{5}$ \\
\hline $\mathrm{V}$ & $4 \times 10^{2}$ & $1 \times 10^{4}$ & $1 \times 10^{4}$ & $6.5 \times 10^{4}$ \\
\hline
\end{tabular}

aithin mapped area. 
TABLE 4

Estimated Parameters of Sources in NGC 6334-I

\begin{tabular}{lcccr}
\hline Source & L(L $\left.\mathrm{L}_{\Theta}\right)$ & $\begin{array}{l}\text { ZAMS }^{\mathrm{a}} \\
\text { Type }\end{array}$ & $\begin{array}{c}\text { Radio }_{(\mathrm{Jy})} \mathrm{F}_{\nu} \\
\text { ZAMS }^{\mathrm{a}} \\
\text { Type }\end{array}$ \\
\hline IRS-1 & $\leq 8 \times 10^{4} \mathrm{~L}_{\Theta} \leq 07.5$ & 1.2 & B0 \\
IRS-2 & $\geq 3 \times 10^{4} \mathrm{~L}_{\Theta} \geq 09.5$ & $<0.02$ & $\leq \mathrm{B} 1$ \\
IRS-3 & $\sim 1 \times 10^{4} \mathrm{~L}_{\Theta}$ & $\sim \mathrm{B} 0.5$ & $<0.02$ & $<\mathrm{BI}$ \\
IRS-4 & $\sim 4 \times 10^{4} \mathrm{~L}_{\Theta}$ & $\sim 09.5$ & 12 & 07.5 \\
\hline
\end{tabular}

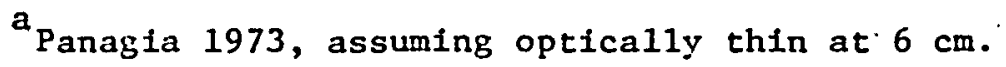
b RCM. 
TABLE 5

Estimated Parameters of SSources in NGC 6334-IV

\begin{tabular}{|c|c|c|c|c|}
\hline Source & $\mathrm{L}\left(\mathrm{L}_{i \theta}\right)$ & $\begin{array}{l}\text { ZAMS }^{\mathrm{a}} \\
\text { Type }\end{array}$ & $\underset{(\mathrm{Jy})}{\operatorname{Radio} F_{v}^{b}}$ & $\begin{array}{l}\text { ZAMs } \\
\text { Type }\end{array}$ \\
\hline IRS-I & $\leq 2 \times 10^{4}$ & $<\mathrm{BO}$ & $<. .02$ & $<\mathrm{BI}$ \\
\hline 2 & $8 \times 10^{4}$ & 07.5 & 10 & $07 . .5$ \\
\hline 3 & $8 \times 10^{4}$ & $07 . .5$ & $<.02$ & $<! \mathrm{B} 1$ \\
\hline 4. & $\leq 2 \times 10^{4}$ & $<\mathrm{BO}$ & $<.02$ & $<\mathrm{BI}$ \\
\hline
\end{tabular}


TABLE 6

Near-Infrared Sources Found in NGC 6334-V

\begin{tabular}{|c|c|c|c|c|c|c|c|}
\hline IRS & RA & & & Dec & & $\mathrm{K}^{\prime}(\mathrm{mag})$ & $K-L$ (mag) \\
\hline 1 & $17^{\mathrm{h}} 16^{\mathrm{m}}$ & $35.0^{5}$ & $-35^{\circ}$ & $54^{\prime}$ & $15^{\prime \prime}$ & 6.32 & 0.55 \\
\hline 2 & & 35.5 & & & 36 & 8.63 & 1.48 \\
\hline 3 & & 36.2 & & & 50 & 10.14 & 1.91 \\
\hline 4 & & 36.6 & & & 59 & 7.44 & 1.47 \\
\hline 5 & & 37.0 & & & 19. & 7.57 & 0.58 \\
\hline 6 & & 38.9 & & & 28 & 7.04 & 1.67 \\
\hline
\end{tabular}

Positional uncertainties are $\pm 7 "$. 


\section{REFERENCES}

Becklin, E. E. et al. 1976, Ap. J., 207, 770.

Becklin, E. E., and Neugebauer, G. (BN) 1974), H II Regions and the Galactic

Center, ed. A. F. M. Moorwood (Neuilly-sur-Seine: ESRO), p. 34.

Bodenheimer, P., Tenorio-Tagle, G., and Yorke, H. W. 1979, Ap. J., 233, 85. Cheung, I., Frogel, J. A., Gezari, D. Y., and Hauser, M. G. 1978, Ap. J.

(Letters), . 226, L149.

Dickel, H. R., Dickel, J. R., and Wilson, W. J. 1977, Ap. J., 217, 56.

Emerson, J. P., Jennings, R. E., and Moorwood, A. F. M. 1973, Ap. J., 184, 401.

Fischer, J., Joyce, R. R., Simon, M., and Simon, T. 1982, Ap. J., 258, 165.

Gatley, I., Becklin, E. E., Sellgren, K., and Werner, M. W. 1979, Ap. J., $\underline{233}, 575$.

Georgelin, Y. M., and Georgelin, Y. P. 1976, Astron. Ap', 49, 57.

Genze1, R., and Downes, D. 1977, Astr. Ap. Supp1., 30, 145.

Gezari, D. Y. 1982, Ap. J., in press.

Harvey, P. Y. 1979, Pub. Astr. Soc. Pac., 91, 143.

Harvey, P. Y., Campbell, M. F., and Hoffmann, W. F. 1978, Ap. J., 219, 891.

Harvey, P. M., Gatley, I., Thronson, H. A., Jr:, and Werner, M. W. 1982,

Ap. J., 258, 568.

Hoffmann, W. F., Frederick, C., and Emery, R. J. 1971, Ap. J. (Letters), 170, L89.

Hyland, A. R., McGregor, P. J., Robinson, G., Thomas, J. A., Becklin, E. E., Gatley, I., and Werner, M. W. 1980, Ap. J., 241, 704.

Icke, V. 1979, Astron. Astrophys., 78, 352.

Icke, V., Gatley, I., , and-Israel, F.P. 1980, Ap. J., .236, 808. 
McBreen, B., Fazio, G. G., Stier, M., and Wright, E. L. (MFSW) 1979, Ap. J. (Letters), 232, L183.

Moran, J. M., and Rodriguez, L. 1980, Ap. J. (Letters), 236, L159.

Neckel, T. 1978, Astron. Ap., 69, 51.

Olthof, J., and van Duinen, R. J. 1973, Astron. Astrophys., 29, 315.

Panagia, N. 1973, A. J., 78, 929.

Raimond, E., and Eliasson, B. 1969, Ap. J., 155, 817.'

Rodriguez, L. F., Canto, J., and Moran, J. M. (RCM) 1982, Ap. J., in press.

Rodriguez, L. F., Moran, J. M., Ho, P. T. P., and Gottlieb, E. W. 1980, Ap. J., 235, 845 .

Schram1, J.,; and Mezger, P. G. 1969, Ap. J., 156, 269.

Simon, M., Righini-Cohen, G., Felli, M., and Fischer, J. 1981, Ap. J., 245, 552.

Wynn-Williams, C. G. 1982, Ann. Rev. Astr. Ap., in press.

Zuckerman, B. 1973, Ap. J., 183, 863. 


\section{FIGURE CAPTIONS}

Figure 1. - Far-infrared contour map of NGC 6334. made by McBreen et al. (1979)... showing the areas observed in this study (dashed lines):

Figure 2 - Infrared maps of NGC 6334-I

(a) $50 \mu \mathrm{m}$. surface brightness contours at levels .025, .05, .1, .2, .3 , and .4 times the peak which is $4.0 \times 10^{-15} \mathrm{Wm}^{-2} \mathrm{~Hz}^{-1} \mathrm{sr}^{-1}$. The cross-hatched area represents the compact core above 0.5 . times the peak. The signal-to-noise was typically 5:1 at the lowest contour.

(b) $100 \mu \mathrm{m}$ surface brightness contours at levels .05, .1,..2, .3, and .4 times the peak which is $4.0 \times 10^{-15} \mathrm{Wm}^{-2} \mathrm{~Hz}^{-1}, \mathrm{sr}^{-1}$. The cross-hatched area represents the compact core above 0.5 times the peak. The signal-to-noise was better than 10:1 at all contour levels.

(c) Dust temperature contours, determined as described in the text; at levels $28,32,36,40,44,48$ and $52 \mathrm{k}$ with a relative uncertainty of $\pm 1.5 \mathrm{~K}(2 \sigma)$.

(d) Dust optical depth contours $\left(\times 10^{-2}\right)$ at $100 \mu \mathrm{m}$ determined as described in the text. The relative uncertainty is $\pm 20 \%(2 \sigma)$.

(e) $20 \mu \mathrm{m}$ surface brightness contours mapped with an 8 " beam at levels $.025, .05, .1, .2, .3$ and .4 times the peak which is $3.5 \times 10^{-15} \mathrm{Wm}^{-2} \mathrm{~Hz}^{-1} \mathrm{sr}^{-1}$. The cross-hatched area is the compact core above 0.5 times the peak. The signal-to-noise was typically 5:1 or better at the lowest contour. 
(f) $20 \mu \mathrm{m}$ surface brightness contours at levels $.05, .1, .2, .3$, $.4, .5, .6, .7, .8, .9$ and 1.0 times $7.2 \times 10^{-15} \mathrm{Wm}^{-2} \mathrm{~Hz}^{-1} \mathrm{sr}^{-1}$ as mapped with a 4" beam. Signal-to-noise as in (e).

(g) $30 \mu \mathrm{m}$ surface brightness contours at levels .05, .1, .2, .3, $.4, .5, .6, .7, .8, .9$ and 1.0 times $1.2 \times 10^{-14} \mathrm{Wm}^{-2} \mathrm{~Hz}^{-1} \mathrm{sr}^{-1}$ as mapped with a 4 " beam. Signal-to-noise as in (e).

Figure 3 - Energy distributions of infrared sources in NGC 6334 labeled by beam size of the measurement or size of area over which the flux was integrated. The $2.2 \mu \mathrm{m}$ data from Becklin and Neugebauer (1974) are labeled "BN."

Figure 4 - Infrared maps of NGC 6334-IV

(a) $50 ; m$ surface brightness contours at levels .2, .3, .4, .5, $.6, .7, .8, .9$ and 1.0 times $1.2 \times 10^{-15} \mathrm{Wm}^{-2} \mathrm{~Hz}^{-1} \mathrm{sr}^{-1}$. The signal-to-noise was better than $10: 1$ at the lowest contuur.

(b) $100 \mu \mathrm{m}$ surface brightness contours at levels .2, .3, .4, .5, $.6, .7, .8, .9$ and 1.0 times $1.7 \times 10^{-15} \mathrm{Wm}^{-2} \mathrm{~Hz}^{-1} \mathrm{sr}^{-1}$. Signal-to-noise as in (a).

(c) Dust temperature contours as in Figure $2 c$ at levels 40,44 and $48 \mathrm{~K}$ with a relative uncertainty of $\pm 1.5 \mathrm{~K}(2 \sigma)$.

(d) Dust optical depth contours at $100 \mu \mathrm{m} \times 10^{-2}$ with relative uncertainty of $\pm 20 \%(2 \sigma)$.

(e) $20 \mu \mathrm{m}$ surface brightness contours as mapped with an $S$ " beam at levels $4,8,12, \ldots, 40 \times 10^{-16} \mathrm{Wm}^{-2} \mathrm{~Hz}^{-1} \mathrm{sr}^{-1}$. The crosshatched area represents a point source of $65^{\circ} \mathrm{Jy}$. The signalto-noise was better than $2: 1$ at the lowest contour. 
(f) 2.2 um surface brightness contours at levels 1, 2, 4, 6, 8 and $10 \times 10^{-20} \mathrm{Wm}^{-2} \mathrm{~Hz}^{-1}: \mathrm{sr}^{-1}$ as mapped with a $10^{\prime \prime}$ beam. The cross-hatched area represents a point source of $.062 \mathrm{Iy}$. The signal-to-noise was better than $3: 1$ at the lowest contour.

Figure 5 - Infrared maps and scans of NGC 6334-V

(a) and (b) Scans (points) relative to beam profiles (dashed lines) at 50 and $100 \mu \mathrm{m}$ in R.A.

(c) $20 \mu \mathrm{m}$ surface brightness contours at levels .05,.1,.2, .3, $.4, \ldots, .9,1.0 \times 1.65 \times 10^{-15} \mathrm{wm}^{-2} \mathrm{~Hz}^{-1} \mathrm{sr}^{-1}$ mapped with an 8 " beam. The signal-to-noise was better than $2: 1$ at the lowest contour.

(d) $20 \mathrm{Hm}$ surface brightness contours at levels .05,.1, .2, .3, $\ldots, .9,1.0 \times 2.8 \times 10^{-15} \mathrm{Wm}^{-2} \mathrm{~Hz}^{-1} \mathrm{sr}^{-1}$ mapped with a: 4" beam and a signal-to-noise better than $3: 1$ at the lowest contour.

(e) $30 \mathrm{im}$ surface brightness contours at levels .05, .I, .2, .3, $\ldots, .9,1.0 \times 1.2 \times 10^{-14} \mathrm{Wm}^{-2} \mathrm{~Hz}^{-1} \mathrm{sr}^{-1}$ mapped with $34 "$ beam and a signal-to-noise better than $2: 1$ at the lowest contour.

(f) Normalized scans at 10 and $20 \mu \mathrm{m}$ with $8^{\prime \prime}$ beam in $R . \dot{\text {. }}$. through declination of peak $20 \mu \mathrm{m}$ surface brightness. Typical noise at $20 \mathrm{pm}$ is comparable to diameter of points.

(g) Normalized jeans at 10, 20 and $30 \mathrm{~km}$ in Dec. with "4" beam and positional accuracy of $20 \mathrm{um}$ through R... UE peak $20 \mu \mathrm{m}$ suriace brightness. The noise levels are $\pm 7 \%, \pm 1.5 \%$, and $53 \%$ of the peak respectively at 10,20 and $30 \mu \mathrm{m}$. 
AUTHOR POSTAL ADDRESSES

\author{
Ian Gatley \\ UKIRT \\ 900 Leilani Street \\ Hilo, HI 96720 \\ Paul M. Harvey \\ Astronomy Department \\ University of Texas \\ Austin, TX 78712
}




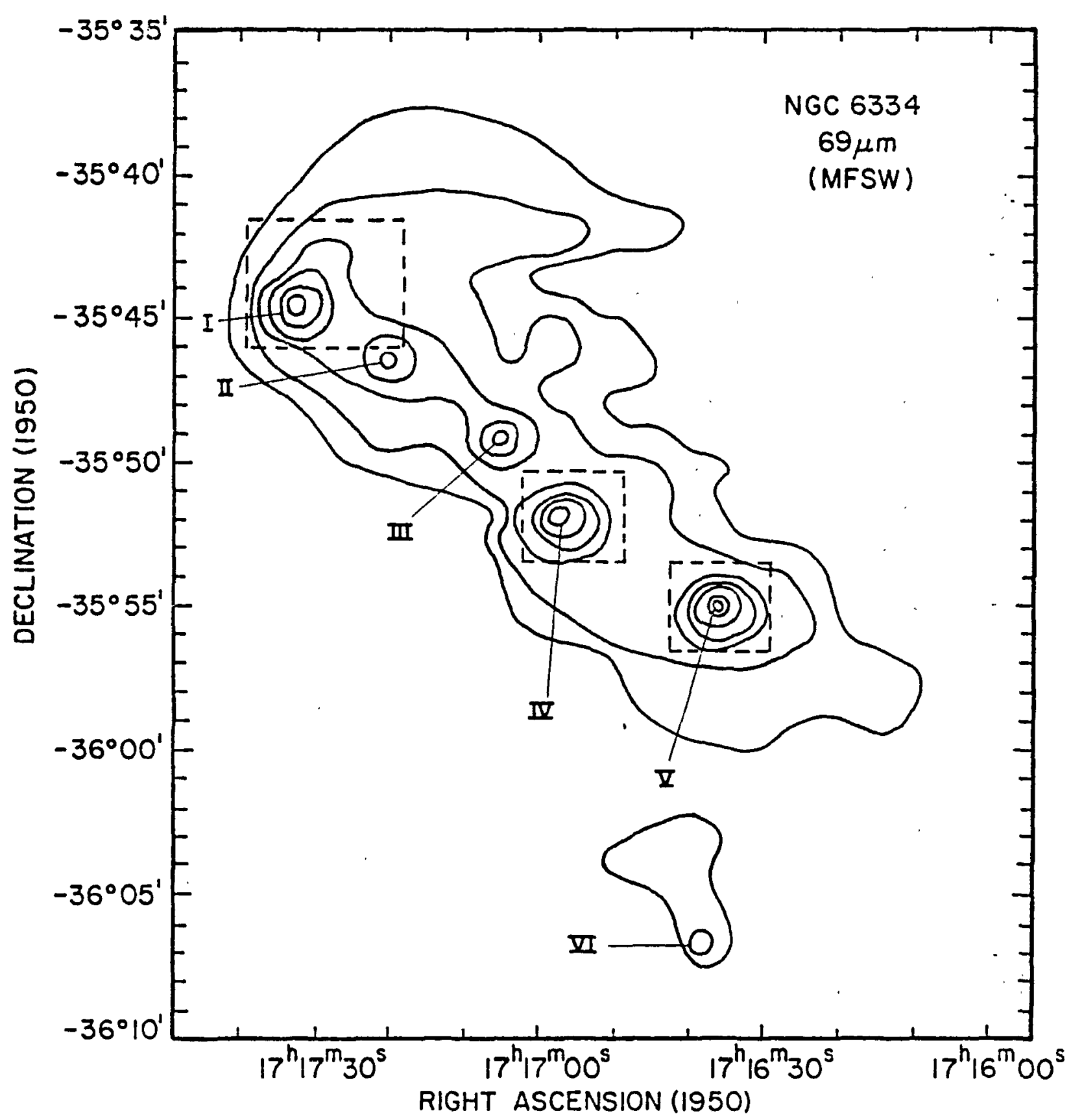

FIGURE L 

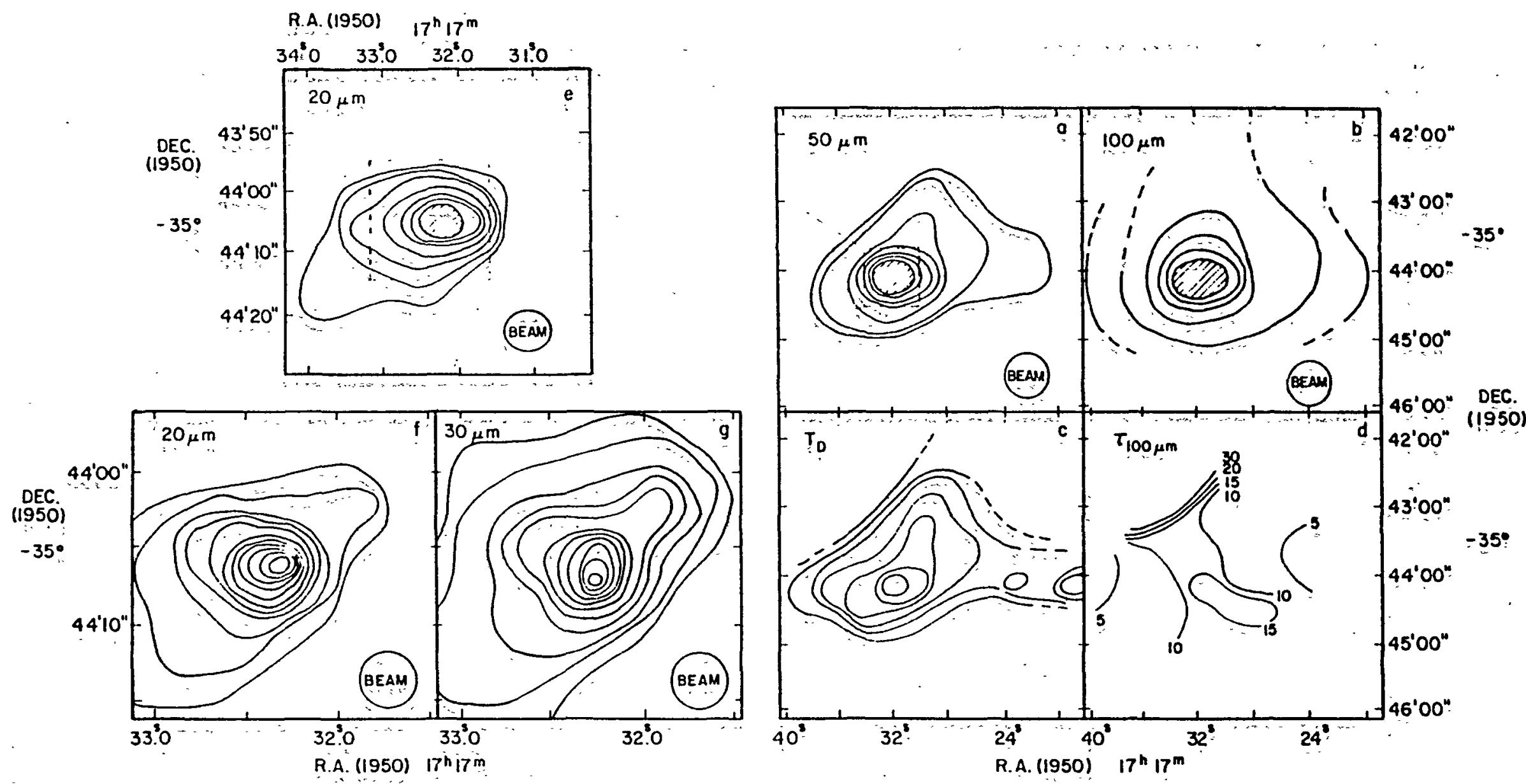

FIGURE 2 


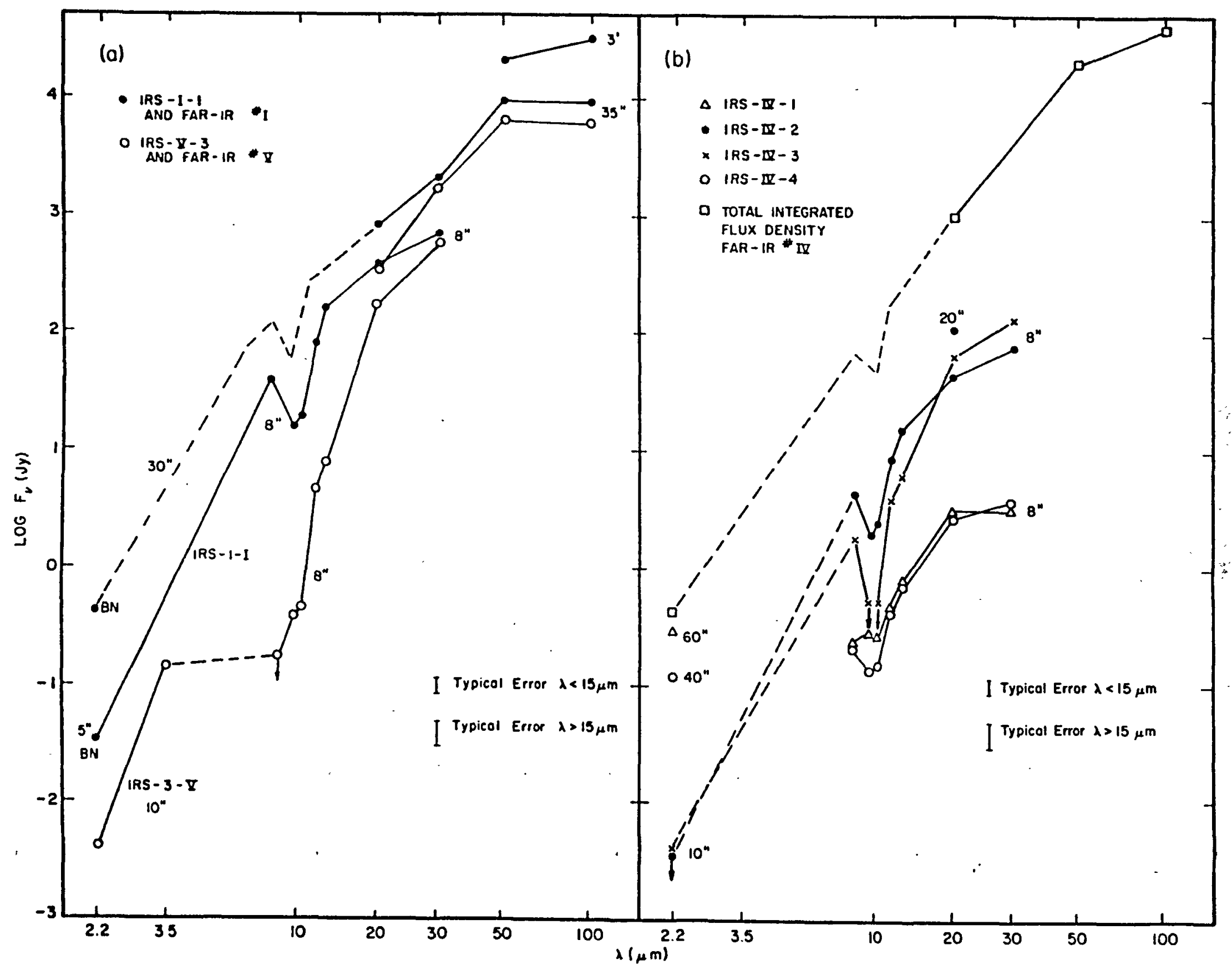

FIGURE 3 

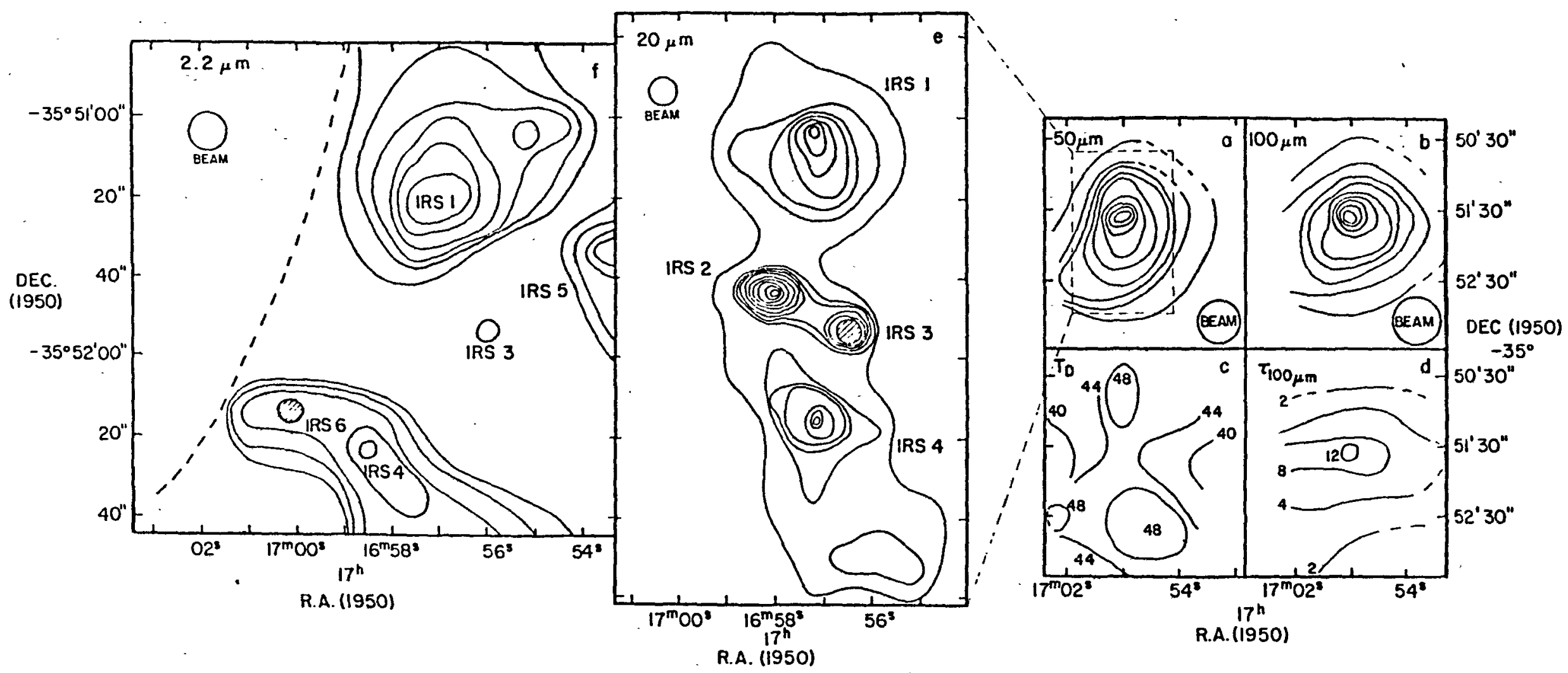

FIGURE 4 


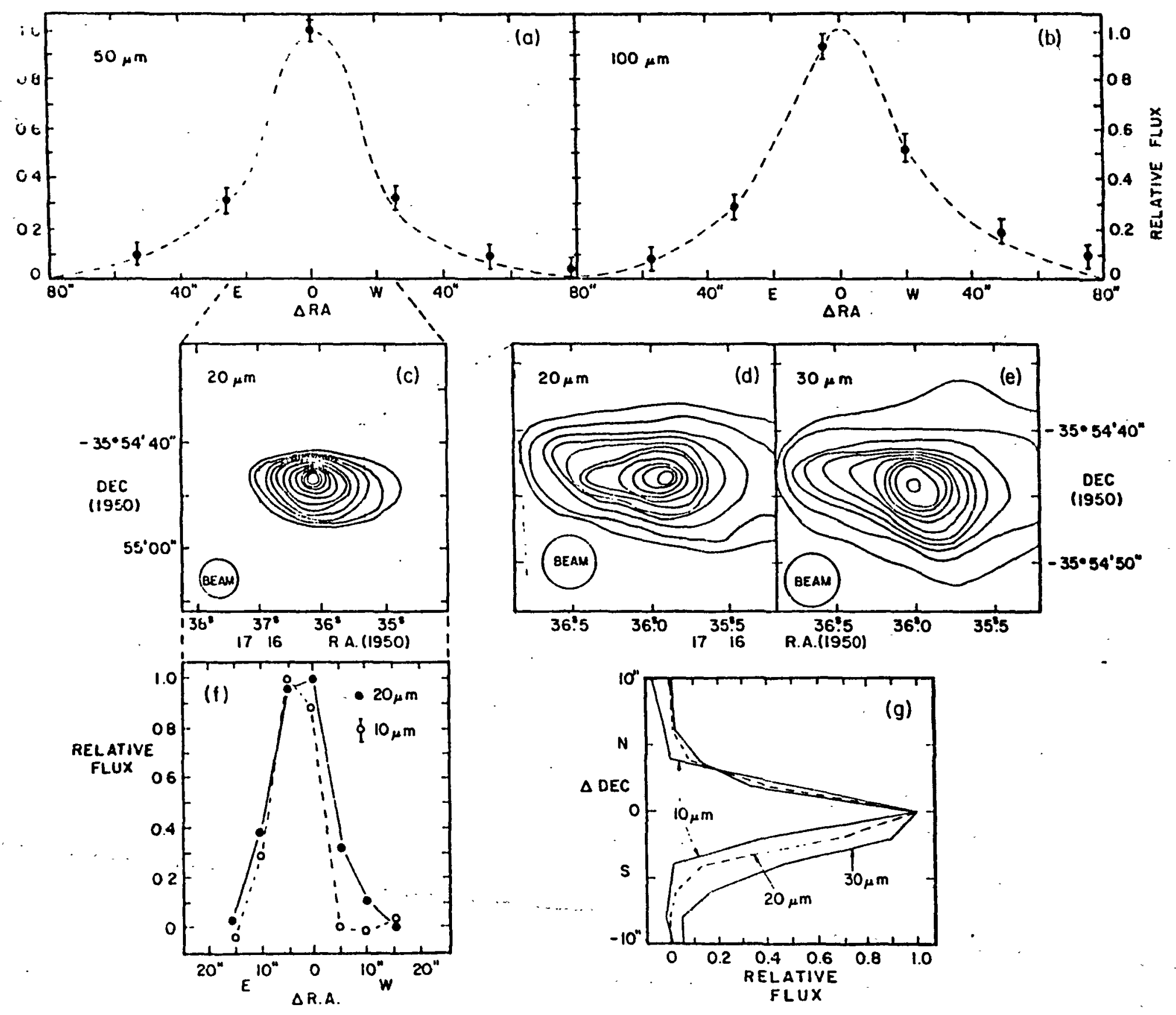




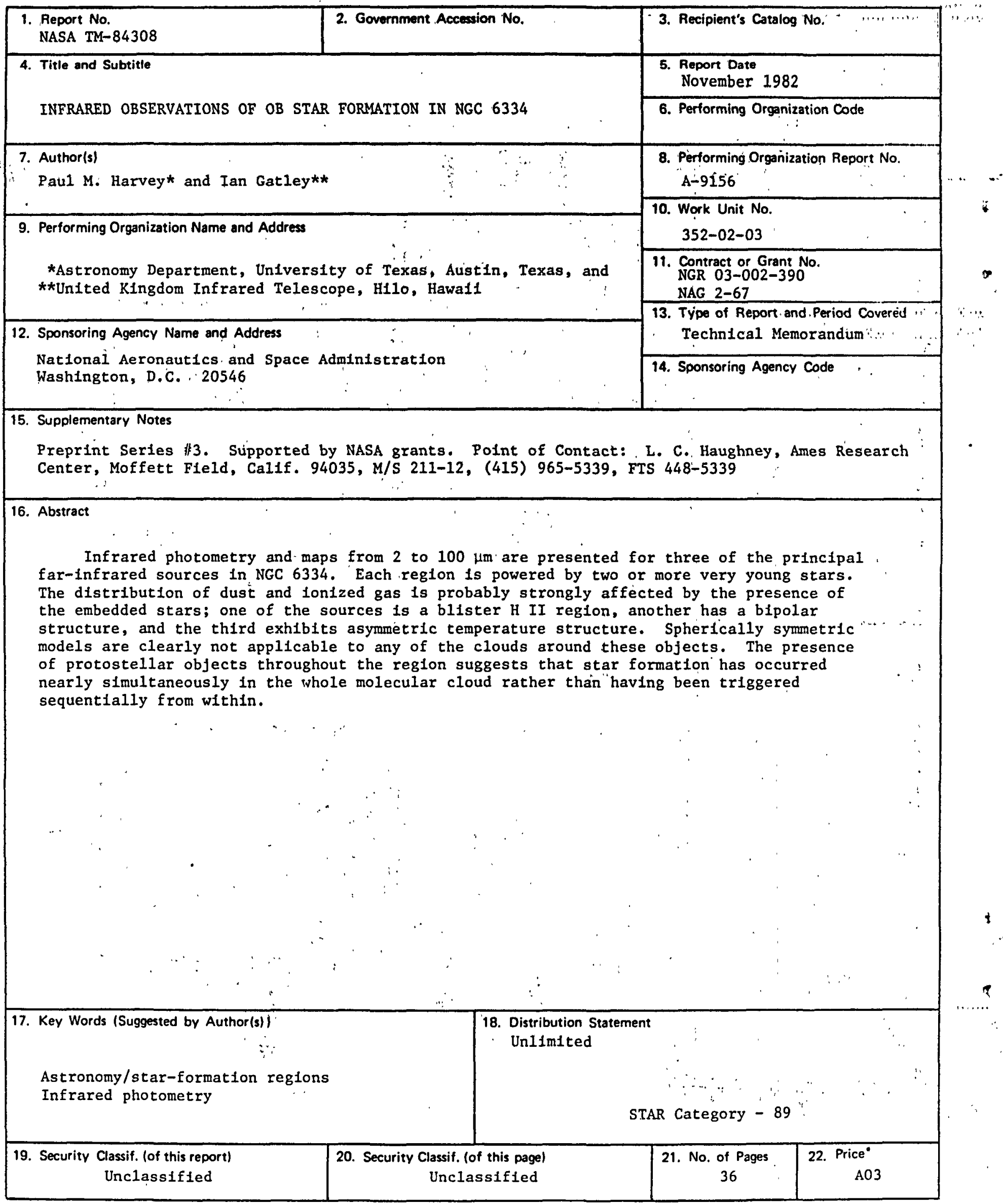

